\section{Antibiotische Therapie der Pneumonie - Was gibt es bei CAP, HAP und VAP zu bedenken?}

\author{
T. Welte ${ }^{1}$ \\ ${ }^{1}$ Direktor der Klinik für Pneumologie, Medizinische Fachhoch- \\ schule Hannover
}

Pneumonien werden aufgrund ihres unterschiedlichen Erregerspektrums und der sich daraus ergebenden unterschiedlichen diagnostischen und therapeutischen Erfordernissen in drei verschiedene Entitäten eingeteilt: ambulant erworbene Pneumonien (community acquired pneumonia, CAP), nosokomiale Pneumonien (hospital acquired pneumonia, HAP) mit der Sonderform der beatmungsassoziierten Pneumonie (ventilator associated pneumonia, VAP) und Pneumonien bei Immunsupprimierten. Bei letzteren spielen neben den bei CAP und HAP beschriebenen bakteriellen Erregern auch opportunistische Infektionen wie Pneumocystis jirovecii, Pilzinfektionen wie die invasive Aspergillose oder Virusinfektionen wie beispielsweise die CMV-Pneumonitis ein Rolle, die in anderen Beiträgen dieses Symposiums besprochen werden. Unter CAP werden alle Infektionen verstanden, die außerhalb des Krankenhauses oder innerhalb der ersten zwei Tage nach Krankenhausaufnahme auftreten. Nach einem Krankenhausaufenthalt sprechen wir nach einer Woche wieder von einer ambulant erworbenen Infektion, wobei in Einzelfällen auch später als eine Woche nach Krankenhausentlassung noch nosokomiale Infektionen auftreten können. Dies ist abhängig von der Länge des Krankenhausaufenthaltes, der Schwere der Erkrankung, des aktuellen Gesundheitszustands des Patienten (Bettlägerigkeit) und der Länge und Häufigkeit der Antibiotikatherapie während des Aufenthaltes. Alle anderen Infektionen werden als HAP bezeichnet; Infektionen, die während einer Beatmung auftreten, werden als VAP gesondert betrachtet.

In Deutschland orientiert sich die Diagnose und Behandlung der Pneumonie an Evidenz-basierten Leitlinien, die für CAP [1] und HAP [2,3] gesondert erstellt wurden.

\section{Die ambulant erworbene Pneumonie $\nabla$}

Der wesentliche Erreger der ambulant erworbenen Pneumonie ist weltweit S. pneumoniae (Pneumokokken), gefolgt von Haemophilus influenzae und Mycoplasma pneumoniae, wobei letzterer epidemisch auftreten kann: Es gibt Jahre mit bis zu $20 \%$ aller Pneumonien infolge von Mykoplasma und Jahre ohne Mykoplasmenpneumonien. Von Mykoplasmen betroffen sind eher jüngere Patienten. Neben diesen häufigen Erregern müssen seltenere Erreger wie Legionella pneumoniae, Staphylococcus aureus, Enterobacteriacae (vor allem Klebsiella pneumoniae) und sehr selten Pseudomonas aeruginosa berücksichtigt werden. Betroffen sind hier vor allem ältere Menschen mit Vorerkrankungen; insbesondere chronisch destruierende Lungenerkrankungen wie die COPD oder die interstitielle Lungenerkrankung stellen einen Risikofaktor dar.
In Einzelfällen gibt es Viruspneumonien (Influenza, RS-Virus, spezielle Coronaviren - SARS und MERS). Die wesentliche Bedeutung der Virusinfektion der oberen Atemwege liegt jedoch darin, dass bakterielle Infektionen durch die Virus-induzierte Immunantwort und Epithelschädigung begünstigt werden und in ihrem Verlauf schwerer (invasiver) zu sein scheinen.

Die Therapie der CAP erfolgt risikostratifiziert. Der CRB-65 Score $(C=$ neu aufgetretene Verwirrtheit, $\mathrm{R}=$ Atemfrequenz $>30 /$ Minute, $\mathrm{B}=$ systolischer Blutdruck $<90 \mathrm{mmHg}$ und Alter $>65$ ) stellt das wesentliche Stratifizierungsinstrument dar. Bei einem Score von 0 kann in der Regel ambulant behandelt werden, bei einem Score von $\geq 2$ sollte der Patient stationär aufgenommen werden. Bei einem Score von 1 müssen zusätzliche Parameter in die Entscheidung zur Hospitalisierung einfließen. Dazu gehören chronische Vorerkrankungen und klinische Parameter wie Hypoxie, Hyper-/Hypokapnie und das Ausmaß der Infiltration im Röntgenbild.

Ein Röntgenthoraxbild und die Bestimmung von Entzündungsparametern gehören zur Basisdiagnostik der CAP. Eine mikrobiologische Diagnostik wird im ambulanten Bereich nicht empfohlen, bei hospitalisierten Patienten sollten zwei Blutkulturpärchen abgenommen werden, bei schweren Infektionen sollte eine Legionelleninfektion durch Bestimmung des Antigens im Urin ausgeschlossen werden. Eine Untersuchung des Atemwegsmaterials ist bei Risikopatienten und Therapieversagern notwendig, vor allem wenn bereits eine antibiotische Vortherapie stattgefunden hat.

Im ambulanten Bereich wird primär eine Therapie mit Amoxicillin über 5 bis maximal 7 Tage empfohlen; als Alternative stehen Makrolidantibiotika und Doxycyclin zur Verfügung. In der Therapie hospitalisierter Patienten (in der Regel intravenös zu Beginn) kommen Ampicillin/Inhibitorkombinationen oder Zweit- bzw. Dritt-Generation-Cephalosporine zum Einsatz. Bei schwerer Pneumonie soll das Betalaktamantibiotikum mit einem Makrolid kombiniert werden, um atypische Erreger empirisch mit zu erfassen; möglicherweise gibt es auch einen positiven immunmodulierenden Effekt der Makrolide. Alternativ können respiratorische Fluorchinolone (Moxifloxacin, Levofloxacin) eingesetzt werden, die Therapie der Wahl für Legionellenpneumonien sind.

Bei Patienten mit Risikofaktoren für eine Infektion mit Staph. aureus (nach Influenza, Aspiration) oder eine komplizierte Infektion mit gram-negativen Erregern (chronische Lungenerkankung, Antibiotikavorbehandlung, Ernährung über Magensonde) sollte primär ein Pseudomonas-aktives Antibiotikum wie Piperacillin/Tazobactam, Ceftazidim oder Meropenem/Impenem zum Einsatz kommen. Auch bei schwer CAP sind 7 Tage Therapie in der Regel ausreichend (Ausnahme: Legionellen, Pseudomonas).

Häufigkeit und Schwere einer Pneumonie können durch Impfmaßnahmen reduziert werden. Etabliert sind die jährliche Influenzaimpfung und die Pneumokokkenimpfung, wobei ein protektiver Effekt auf Pneumonien nur für den 13valenen Konjugatimpfstoff nachgewiesen wurde. 
Nosokomiale Pneumonie (HAP) einschließlich beatmungsassoziierter Pneumonie

$\nabla$

Die wesentlichen Erreger der nosokomialen Pneumonie sind Staphylococcus aureus, Enterobacteriacae (E. coli, K. pneumoniae) und Non Fermenter (P. aeruginosa, Acinetobacter species). Aspergillusinfektionen kommt auch bei immunkompetenten Patienten eine zunehmende Bedeutung zu, vor allem bei COPD-Patienten mit oraler oder hochdosierter inhalativer Corticosteroidtherapie. Candida stellt im Atemwegsmaterial praktisch immer einen Kolonisationskeim dar und muss nicht behandelt werden.

Während bei HAP überwiegend mit sensiblen Erregern zu rechnen ist, muss bei VAP in Abhängigkeit von der antibiotischen Vortherapie und der Länge der Beatmung mit resistenten Erregern gerechnet werden. Durch die Zunahme des Alters und der Multimorbidität von Patienten verschwimmen die Unterschiede jedoch zunehmend.

Basisdiagnostik bleiben das Röntgenbild und die Entzündungsparameter, aufgrund der Multimorbidität und eingeschränkter Qualität (Röntgenbild) ist die Differenzialdiagnose schwierig. Eine mikrobiologische Diagnostik (Blutkulturen, Atemwegsmaterial) sollte daher bei HAP/VAP angestrebt werden. Im Einzelfall (z.B. Aspergillusinfektion) ist eine erweiterte Diagnostik (CT-Thorax, Galatomannan in der BAL) notwendig.

Die Therapieempfehlung orientiert sich am Risiko des Patienten für multiresistente Erreger. Bei sensiblen Erregern ist eine Monotherapie mit Beta-Laktamantibiotika möglich, nach Erregeridentifikation kann entsprechend des Antibiogramms auf die entsprechende Substanz deeskaliert werden. Bei Risikofaktoren für multiresistente Erreger wird - vor allem bei schweren Infektionen - eine Kombinationstherapie aus Pseudomonas wirksamem Beta-Laktam und Aminoglykosid (einmal täglich hochdosiert, Spiegelkontrolle empfohlen) oder Fluorchinolonen empfohlen. Auch hier kann nach Erhalt des Antibiogramms auf eine Monotherapie deeskaliert werden. Die Therapiedauer sollte auch bei HAP/VAP sieben Tage betragen. Für bestimmte Erreger (Pseudomonas, Aspergillus) und bestimmte Infektionen (Lungenabszess, Pleuraempyem) gelten längere Behandlungsempfehlungen.

Für resistente Erreger gibt es spezifische Therapieempfehlungen. Für MRSA wird Linezolid, für gegen alle Basissubstanzen resistente Non Fermenter (4MRGN) Colistin oder Fosfomycin, für Carbapenem-resistene Erreger Colistin oder Tigecyclin empfohlen. Die Rolle von inhalativen Antibiotika für die Therapie multiresistenter Erreger, insbesondere bei VAP, ist umstritten.

Aufgrund des in der Regel höheren Herzminutenvolumens von Intensivpatienten, des durch Kapillarleck veränderten Verteilungsvolumens und der aufgrund der Hypalbuminämie veränderten Plasmaeiweißbindung sind die Antibiotikaspiegel in der Intensivtherapie häufig reduziert. Es soll immer entsprechend der maximalen empfohlenen Dosis therapiert werden; Spiegelbestimmungen sind, wenn vorhanden (Aminoglykoside, Glykopeptide, Voriconazol) empfohlen. Mit modernen, auf der Intensivstation verfügbaren Dialyseverfahren werden die meisten Antibiotika annähernd normal eliminiert. Dosisreduktionen, wie bei chronischer Hämodialyse empfohlen, sind daher in der Regel nicht notwendig. Zunehmend werden mehr Untersuchungen zur Dosierung unter Intensivbedingungen publiziert, an denen man sich orientieren sollte.

Spezifische Impfungen für die wichtigsten HAP/VAP-Erreger gibt es bisher nicht. Pseudomonas- und Staphylokokkenimpstoffe befinden sich jedoch zurzeit in klinischen Studien der Phase II/III.

Seit einem Jahrzehnt gibt es erstmals wieder wesentliche Antibiotika- (und Antimykotika-) Neuentwicklungen. Ceftobiprol [4], ein dem Ceftazidim im Wirkungsspektrum ähnliches, jedoch MRSA-wirksames Fünftgenerationscephalosporin ist seit 30.09.2014 in Deutschland erhältlich.

Mit Tedizolid, einem dem Linezolid ähnlichen Oxazolidinon mit deutlich höherer Bakterizidie, dem Betalaktamaseinhibitor Avibactam, der gegen die meisten Breitspektrumbetalaktamasen und Carbapenemasen aktiv ist, und dem gegenüber Pseudomonas extrem wirksamen Ceftolozane befinden sich Antibiotika mit neuem Wirkmechanismus in Phase-III-Zulassungsstudien.

Bei den Antimykotika wird das bereits oral verfügbare Aspergillus- und Mukor-aktive Posaconazol noch dieses Jahr als intravenöse Zubereitung erhältlich sein. Mit Isavuconazol befindet sich eine weitere Substanz im Zulassungsverfahren. Interessenkonflikte: Tobias Welte ist Mitglied des Advisory Boards von Basilea Pharmaceutica, Bayer, Cubist, Novartis und Pfizer. Er hat Honorare für Vorträge von Astellas, AstraZeneca, Basilea Pharmaceutica, Bayer, GSK, Infectopharm, MSD, Novartis und Pfizer bekommen.

\section{Literatur}

1 Höffken G, Lorenz J, Kern Wet al. Epidemiology, diagnosis, antimicrobial therapy and management of community-acquired pneumonia and lower respiratory tract infections in adults. Guidelines of the Paul-Ehrlich-Society for Chemotherapy, the German Respiratory Society, the German Society for Infectiology and the Competence Network CAPNETZ Germany. Pneumologie 2009; 63: e1-e68

2 Dalhoff $K$, Abele-Horn M, Andreas $S$ et al. Epidemiology, diagnosis and treatment of adult patients with nosocomial pneumonia. S-3 Guideline of the German Society for Anaesthesiology and Intensive Care Medicine, the German Society for Infectious Diseases, the German Society for Hygiene and Microbiology, the German Respiratory Society and the Paul-Ehrlich-Society for Chemotherapy. Pneumologie 2012; 66: $707-765$

3 Dalhoff K, Ewig S; Guideline Development Group. Adult patients with nosocomial pneumonia: epidemiology, diagnosis, and treatment. Dtsch Arztebl Int 2013; 110: 634-640

4 Awad SS, Rodriguez AH, Chuang YC et al. A Phase 3 randomized doubleblind comparison of ceftobiprole medocaril versus ceftazidime plus linezolid for the treatment of hospital-acquired pneumonia. Clin Infect Dis 2014 Apr 9 [Epub ahead of print]

\section{Bibliografie}

Dol http://dx.doi.org/10.1055/s-0033-1358031

Drug Res 2014; 64, Suppl. 1: S11-S12

(c) Georg Thieme Verlag KG Stuttgart · New York .

ISSN 2194-9379

Korrespondenzadresse

Tobias Welte

Medizinische Hochschule Hannover

Carl-Neuberg-Straße 1

30625 Hannover

welte.tobias@mh-hannover.de 